\title{
Parotid Metastasis of Renal Cell Carcinoma: A Case Report and Review of the Literature
}

\author{
Claire M. Lawlor ${ }^{2}$ and Richard O. Wein ${ }^{1,2 *}$ \\ Correspondence: rwein@tuftsmedicalcenter.org \\ ${ }^{1}$ Department of Otolaryngology-Head \& Neck Surgery at Tufts Medical Center \& \\ ${ }^{2}$ Tufts University School of Medicine, Boston, MA.
}

\begin{abstract}
Background: To review the presentation and management of renal cell carcinoma metastatic to the parotid gland. Methods: Case report and review of the literature.

Results: There have been 45 cases of renal cell carcinoma metastatic to the parotid gland reported in the English literature. A parotid lesion can be the initial clinical presentation of this malignancy (even before primary site identification). A case of patient with a parotid mass noted 4 years after undergoing a nephrectomy for renal cell carcinoma is presented. Superficial parotidectomy with facial nerve monitoring demonstrated metastatic renal cell carcinoma with negative margins on pathology.

Conclusions: Metastatic renal cell carcinoma should be considered in the differential diagnosis a vascular parotid mass in patients have a history of prior diagnosis of renal cell carcinoma. After diagnosis is established, a systemic assessment should be performed given that solitary parotid metastases are uncommon. Due to the limited number of cases reported in the literature, clinical outcomes are difficult to predict.
\end{abstract}

Keywords: renal cell carcinoma, parotid metastases, parotidectomy, distant metastasis.

\section{Background}

Malignancies of the parotid gland are relatively uncommon, accounting for only 3-6\% of all head and neck cancers and $0.3 \%$ of all cancers [1]. Approximately $30 \%$ of parotid tumors are malignant and most represent primary salivary gland tumors. Metastatic spread to the parotid is uncommon but should be considered at the time of initial assessment of a parotid mass. The most common primary malignancies associated with metastasis to the parotid are cutaneous neoplasms of the head and neck [2]. However, ductal carcinoma of the breast, rhabdomyosarcoma, small cell carcinoma of the lung, and renal cell carcinoma are also primary tumors that harbor the potential for metastatic spread to the parotid that have been reported $[2,3]$.

Renal cell carcinoma (RCC) is a relatively uncommon cancer with approximately 54,000 new cases and 13,000 deaths noted annually in the United States. It has a 3:2 male predominance and a peak incidence in the $6^{\text {th }}$ and $7^{\text {th }}$ decades of life. Though tobacco use, obesity, and hypertension are the only identified risk factor, most cases are sporadic. The classic triad of clinical presentation includes hematuria, pain, and abdominal mass, however greater than $50 \%$ of cases are detected incidentally during abdominal imaging for another indication [4].

RCC is well known for its unique potential to metastasize to nearly every organ system in the body. The tumor is highly vascular and thought to metastasize via both hematogenous (via the Batson's plexus) and lymphatic routes [5]. The most common sites for metastasis are the lung, bone, adrenal, liver, brain, and the contralateral kidney [4]. Though not as frequent, metastatic RCC to the head and neck has been identified in the thyroid, salivary glands, skull base, sinuses, pharynx, tonsils, tongue, lip and skin [6]. Metastasis to the parotid gland is very rare. In a study by Bernicker et al of 65 patients with RCC metastasis to the head and neck, none of the patients presented with parotid metastasis [6]. The first case of RCC metastatic to the parotid gland was published by Patey et al in 1965 [7]. Since then there have only been 45 reported cases reported in the English literature [5,7-37,39]. Although this entity is uncommon in presentation, this review highlights issues that may assist the head and neck surgeon with a pathologic diagnosis not often encountered cephalad to the clavicles.

\section{Methods}

PubMed and MEDLINE were used to search the English literature database (from 1965-2011) using the terms "renal cell carcinoma" and "parotid gland." References from those articles were thoroughly reviewed. Only relevant publications were included. Cases of renal cell carcinoma metastatic to the parotid gland were reviewed with emphasis on patient age, gender, presenting symptoms and duration, time interval since diagnosis of primary cancer, physical exam, diagnostic tests results including fine needle aspiration (FNA), radiologic 
imaging, pathologic diagnosis, management, other sites of distant metastasis, and postoperative outcome. Finally, the author's case report of renal cell carcinoma metastatic to the parotid gland was retrospectively reviewed with attention to clinic notes, operative notes, radiology, and pathology reports and is presented below.

\section{Results}

\section{Case report}

A 71 year-old man was referred for management of a right parotid mass that was first noticed 2 years prior. Over the preceding 12 months the mass had become increasingly tender after being accidentally traumatized. The patient denied any significant change in size of the mass, paresthesia, or facial weakness. There was no associated dysphagia, hoarseness, otalgia, dyspnea, or unexplained weight loss. He had a past medical history significant for hypertension. Of note, the patient had undergone a right nephrectomy 5 years prior for renal cell carcinoma. The patients also had a 50+ pack-year smoking history and denied a family history of renal diagnoses or other cancer syndromes.

On physical exam, there was a non-tender, soft mass in the tail of the right parotid without associated lymphadenopathy. The patient's body-mass index (BMI) was 24.33 and blood pressure was 145/65 during evaluation. Both computed tomography (CT) scan (Figure 1 \& 2) and magnetic resonance imaging (MRI) of the neck with contrast had been performed prior and showed an isolated right-sided lobular parotid mass that measured $3.0 \times 2.4 \times 2.5 \mathrm{~cm}$. Of note, the mass demonstrated an intense uptake of contrast. On repeat MRI at the time of evaluation there was noted to be an interval increase of the mass to $3.5 \times 3.2 \times 3.7 \mathrm{~cm}$. The patient underwent a total parotidectomy with facial nerve preservation. Intraoperative facial nerve 4-channel monitoring was utilized.

On pathologic review, the parotid was noted to harbor a soft, well-encapsulated, orange-tan, homogenous wellcircumscribed lesion with areas of hemorrhage (Figure 3). The tumor appeared to be confined within a thin capsule. The remainder of the specimen demonstrated normal parotid tissue. Histological evaluation demonstrated polygonal to round cells with abundant clear cytoplasm and large nucleoli containing small nucleoli (Figure 2), in a stark contrast to normal parotid tissue (Figure 4). Immunohistochemistry revealed positive staining for vimentin (Figure 5) and CD10 (Figure 6), which lead to the diagnosis of renal cell carcinoma metastatic to the parotid gland.

Following this diagnosis, the patient underwent full-body imaging which demonstrated 3 small lesions in the pancreas that were stable compared to prior imaging. One year after the surgery, the patient was doing well and remained disease-free. Review of literature.

The English literature reports 45 cases of RCC metastatic to the parotid gland since 1965 [5,7-37,39]. Only 31 complete case presentations have been documented (Table 1).

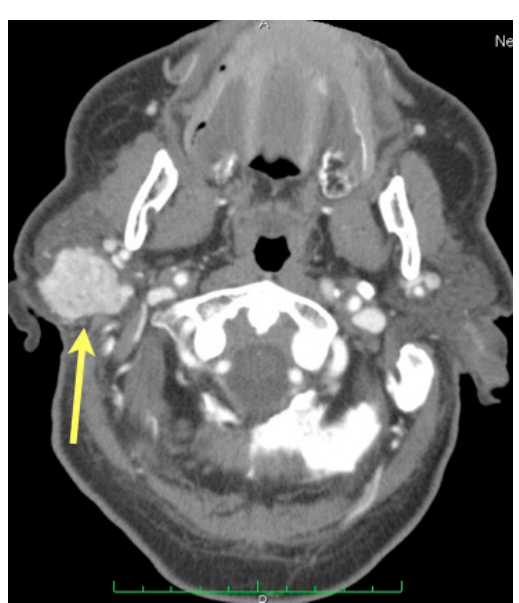

Figure 1. Axial and coronal views of a CT scan with contrast of the neck demonstrating a vascular lesion within the superficial aspect of the right parotid gland.

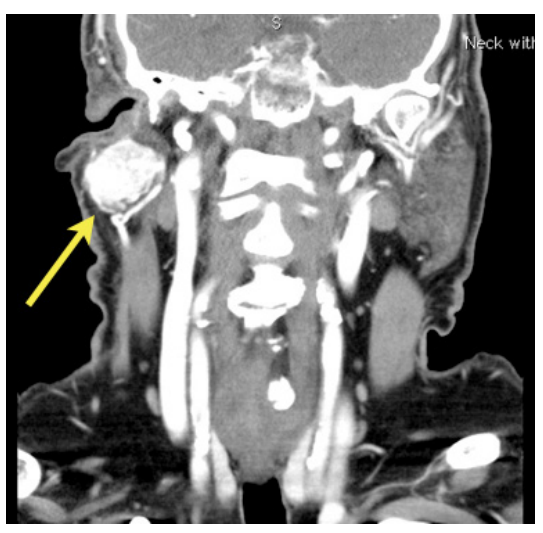

Figure 2. Axial and coronal views of a CT scan with contrast of the neck demonstrating a vascular lesion within the superficial aspect of the right parotid gland.

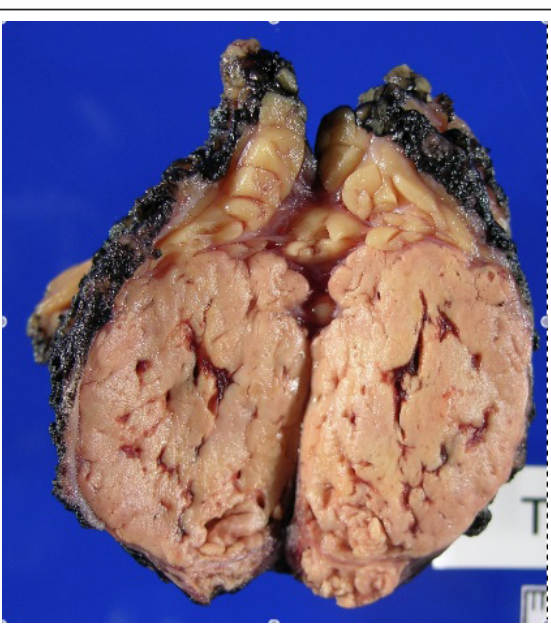

Figure 3. Right superficial parotidectomy gross specimen 


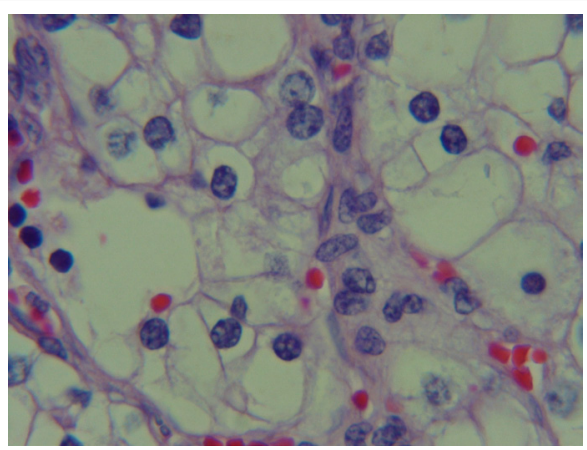

Figure 4. Hematoxylin and eosin stain of carcinoma cells. Note the optically clear cytoplasm and large slightly basophilic nuclei containing small nucleoli.

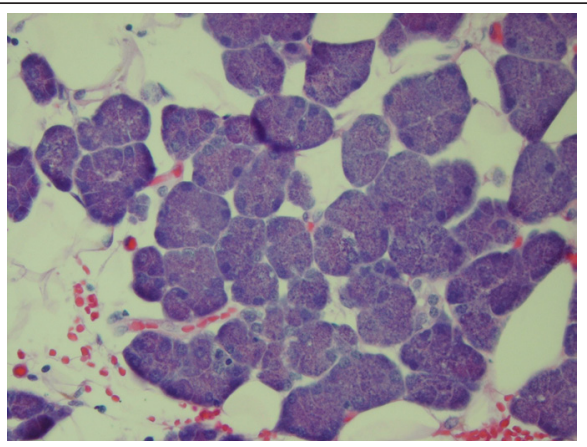

Figure 5. Hematoxylin and eosin stain of normal parotid tissue demonstrating acini and granular cytoplasm.

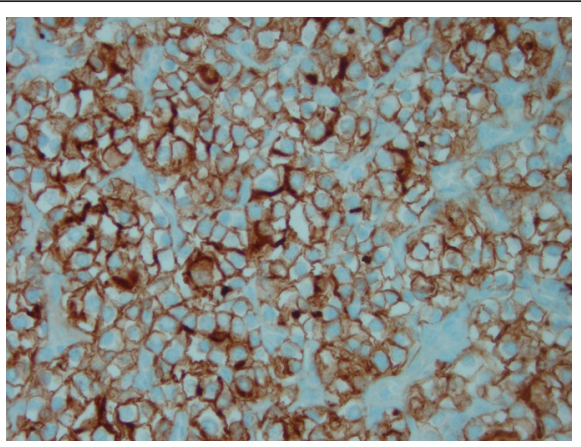

Figure 6. Strongly positive vimentin immunohistochemical stain in carcinoma cells.

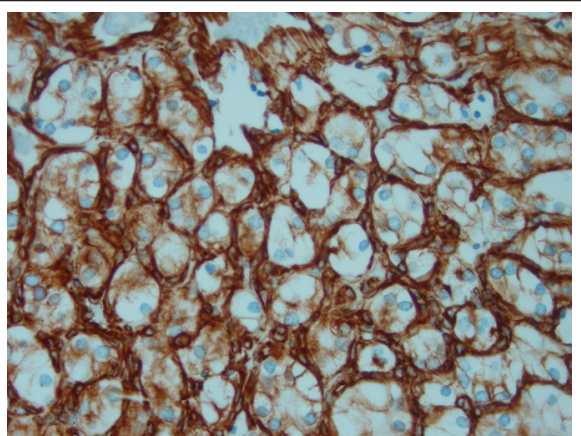

Figure 7. Strongly positive CD10 immunohistochemical stain in carcinoma cells.
[5,7-11,13-17,19,20,22-30,32-35,39]. There was a 2:1 male predominance. Age ranges from 42-83, with a mean of 61.9 $( \pm 9.5)$. Twenty-nine of the patients presented with unilateral parotid metastasis. Sarangi et al. [23] described a patient that initially presented with a left parotid mass and developed a right parotid mass 4 months later. Ravi et al. [22] reported a case of bilateral parotid metastases. All of the patients had a palpable mass on presentation and 19 of the patients were asymptomatic. Associated symptoms included painful or tender masses (7), pulsatile mass (2), bruit over mass (1), tinnitus (1), ipsilateral facial weakness (1), and numbness of the ipsilateral commissure of the lip (1).

In 16 of the cases, parotid metastasis was the first sign of the renal primary. Fourteen of the patients had been diagnosed and treated for RCC in prior to parotid metastasis with an interval prior parotid presentation that spanned from 5 months to 19 years. Wayne et al. [9] reported a patient that was diagnosed with RCC of the parotid but no primary tumor was ever identified. In 16 of the cases, the patients were found to have metastatic disease beyond the parotid upon subsequent work-up. Four patients appeared to have isolated parotid disease at the time of their presentation but went on to develop other distant metastatic disease. There were 4 cases of isolated parotid metastasis. Seven reports did not discuss distant metastasis.

Fine needle aspiration was performed in 13 of the reported cases. It was diagnostic in 3 , non-diagnostic in 10 , and not mentioned in 18 of the published cases. Twenty-eight of the patients underwent excision of the parotid disease. Fifteen patients underwent superficial parotidectomies, 1 underwent a partial parotidectomy, 1 underwent a "deep" parotidectomy, and 5 underwent total parotidectomy. The remaining 6 authors did not detail the extent of the procedure beyond excision of the mass. Three authors elected to perform neck dissections in addition to parotidectomy. 11 authors mentioned the facial nerve with preservation noted in 10 of the cases. In 1 case the inferior portion of the facial nerve required sacrifice for complete resection of the tumor. There were 3 reports of locally recurrent disease after excision. Two patients were not managed with palliative intent with radiotherapy and pain relief. One author did not comment on therapy.

Eight of the articles published since 1989 described immunohistochemical stains used to identify the primary tumor (Table 1) [9,11-14,20,28,39]. RCC consistently stained positive for proximal nephron renal antigen (PNRA), vimentin, CD10, and cytokeratin (CAM5.2). Common negative stains were carcinoembryonic antigen (CEA) and cytokeratin-7.

\section{Discussion}

Metastasis from RCC to the parotid gland is a rare finding. RCC also poses a particular diagnostic challenge because distant disease can present synchronous with the diagnosis of the primary renal tumor or a metachronous distant presentation may occur many years after therapy for the primary. For greater than $50 \%$ of the case presentations reviewed, the parotid lesion was 
Lawlor et al. Journal of Cancer Therapeutics \& Research 2012,

http://www.hoajonline.com/journals/pdf/2049-7962-1-15.pdf

doi: 10.7243/2049-7962-1-15

Table 1. Summary of all complete case presentations of RCC metastatic to the parotid gland dating from 1965 to present. R.: right, L.: left, RCC: renal cell carcinoma, mets: metastasis

\begin{tabular}{|c|c|c|c|c|c|c|c|c|c|c|c|}
\hline Author & Year & Age & Sex & Presenting symptom & $\begin{array}{l}\text { Synchro- } \\
\text { nous or } \\
\text { metachro- } \\
\text { nous }\end{array}$ & $\begin{array}{l}\text { First } \\
\text { sign } \\
\text { of } \\
\text { RCC? }\end{array}$ & RCC & $\begin{array}{l}\text { Other simultaneous } \\
\text { mets? }\end{array}$ & $\begin{array}{l}\text { Fine Needle } \\
\text { aspiration }\end{array}$ & Treatment & $\begin{array}{l}\text { Immunohisto- } \\
\text { chemistry }\end{array}$ \\
\hline $\begin{array}{l}\text { Patey et. } \\
\text { al. }\end{array}$ & 1965 & 63 & $\mathrm{~F}$ & $\begin{array}{l}\text { Pulsatile mass } x 1 \\
\text { year }\end{array}$ & $\begin{array}{l}\text { Metachro- } \\
\text { nous }\end{array}$ & $\mathrm{Y}$ & RCC $d x$ years later & Not discussed & $\begin{array}{l}\text { Not men- } \\
\text { tioned }\end{array}$ & $\begin{array}{l}\text { Radiotherapy and } \\
\text { excision }\end{array}$ & $\begin{array}{l}\text { None men- } \\
\text { tioned }\end{array}$ \\
\hline $\begin{array}{l}\text { Kucan } \\
\text { et. al. }\end{array}$ & 1981 & 55 & M & Mass x3-4mo. on R. & $\begin{array}{l}\text { Synchro- } \\
\text { nous }\end{array}$ & Y & $\begin{array}{l}\text { RCC dx on subsequent } \\
\text { work up }\end{array}$ & Not discussed & $\begin{array}{l}\text { Not men- } \\
\text { tioned }\end{array}$ & $\begin{array}{l}\text { Superficial parotidec- } \\
\text { tomy }\end{array}$ & $\begin{array}{l}\text { None men- } \\
\text { tioned }\end{array}$ \\
\hline $\begin{array}{l}\text { Sist et. } \\
\text { al. }\end{array}$ & 1982 & 62 & M & Mass $x 2$ years on $L$. & $\begin{array}{l}\text { Synchro- } \\
\text { nous }\end{array}$ & Y & $\begin{array}{l}\text { R. RCC dx in subse- } \\
\text { quent work up }\end{array}$ & Not discussed & $\begin{array}{l}\text { Not men- } \\
\text { tioned }\end{array}$ & $\begin{array}{l}\text { Deep parotidectomy } \\
w / \text { preservation of the } \\
\text { facial nerve }\end{array}$ & $\begin{array}{l}\text { None men- } \\
\text { tioned }\end{array}$ \\
\hline $\begin{array}{l}\text { Percival } \\
\text { et. al. }\end{array}$ & 1982 & 71 & $\mathrm{~F}$ & $\begin{array}{l}\text { Mass } \times 3 \text { years } \\
\text { on } R .\end{array}$ & $\begin{array}{l}\text { Synchro- } \\
\text { nous }\end{array}$ & Y & $\begin{array}{l}\text { L. RCC dx one year } \\
\text { later }\end{array}$ & Liver, lung & $\begin{array}{l}\text { Not men- } \\
\text { tioned }\end{array}$ & Surgical excision & $\begin{array}{l}\text { None men- } \\
\text { tioned }\end{array}$ \\
\hline $\begin{array}{l}\text { Smits et. } \\
\text { al. }\end{array}$ & 1984 & 60 & $\mathrm{~F}$ & Painful mass on $\mathrm{R}$. & $\begin{array}{l}\text { Metachro- } \\
\text { nous }\end{array}$ & $\mathrm{N}$ & $\begin{array}{l}\text { R. nephrectomy for } \\
\text { RCC } 8.5 \text { years earlier }\end{array}$ & $\begin{array}{l}\text { Submandibular } \\
\text { gland }\end{array}$ & $\begin{array}{l}\text { Not men- } \\
\text { tioned }\end{array}$ & Excision of mass & $\begin{array}{l}\text { None men- } \\
\text { tioned }\end{array}$ \\
\hline $\begin{array}{l}\text { Hessan } \\
\text { et. al. }\end{array}$ & 1986 & 52 & M & Mass x $2 \mathrm{mo}$. on $\mathrm{L}$. & $\begin{array}{l}\text { Synchro- } \\
\text { nous }\end{array}$ & $\mathrm{Y}$ & $\begin{array}{l}\text { L. RCC dx in subse- } \\
\text { quent work up }\end{array}$ & $\begin{array}{l}\text { None at time of } \\
\text { parotid presenta- } \\
\text { tion, later lungs, } \\
\text { ribs, lumbar spine, } \\
\text { and brain mets. }\end{array}$ & $\begin{array}{l}\text { Not men- } \\
\text { tioned }\end{array}$ & $\begin{array}{l}\text { Superficial parotidec- } \\
\text { tomy }\end{array}$ & $\begin{array}{l}\text { None men- } \\
\text { tioned }\end{array}$ \\
\hline $\begin{array}{l}\text { Harrison } \\
\text { et. al. }\end{array}$ & 1987 & 64 & $\mathrm{~F}$ & $\begin{array}{l}\text { Mass } \times 2 \text { mo.on } \\
\text { R., R. superficial } \\
\text { parotidectomy } \\
4 \text { years prior for } \\
\text { "clear cell tumor" }\end{array}$ & $\begin{array}{l}\text { Metachro- } \\
\text { nous }\end{array}$ & $\mathrm{N}$ & $\begin{array}{l}\text { Nephrectomy for RCC } \\
10 \text { years prior }\end{array}$ & None & $\begin{array}{l}\text { Not men- } \\
\text { tioned }\end{array}$ & Excision of lesions & $\begin{array}{l}\text { None men- } \\
\text { tioned }\end{array}$ \\
\hline \multirow[t]{2}{*}{$\begin{array}{l}\text { Owens } \\
\text { et. al. }\end{array}$} & 1989 & 55 & M & Mass x3mo. on $\mathrm{R}$. & $\begin{array}{l}\text { Synchro- } \\
\text { nous }\end{array}$ & Y & $\begin{array}{l}\text { L. RCC dx in subse- } \\
\text { quent work up }\end{array}$ & Chest, brain, bone & $\begin{array}{l}\text { Not men- } \\
\text { tioned }\end{array}$ & $\begin{array}{l}\text { Superficial parotidec- } \\
\text { tomy w/ partial excision } \\
\text { of masseter }\end{array}$ & $\begin{array}{l}\text { None men- } \\
\text { tioned }\end{array}$ \\
\hline & & 75 & $\mathrm{~F}$ & $\begin{array}{l}\text { Pulsatile mass x10 } \\
\text { weeks on L. }\end{array}$ & $\begin{array}{l}\text { Metachro- } \\
\text { nous }\end{array}$ & $\mathrm{N}$ & $\begin{array}{l}\text { R. nephrectomy for } \\
\text { RCC } 7 \text { years prior }\end{array}$ & $\begin{array}{l}\text { None at time of } \\
\text { parotid presenta- } \\
\text { tion, later recur- } \\
\text { rent renal disease. }\end{array}$ & $\begin{array}{l}\text { Not men- } \\
\text { tioned }\end{array}$ & $\begin{array}{l}\text { Total parotidectomy } \\
w / \text { preservation of the } \\
\text { facial nerve }\end{array}$ & $\begin{array}{l}\text { None men- } \\
\text { tioned }\end{array}$ \\
\hline $\begin{array}{l}\text { Melnick } \\
\text { et. al. }\end{array}$ & 1989 & 72 & M & $\begin{array}{l}\text { Mass } \times 2.5 \text { years } \\
\text { on } \mathrm{L} \text {. }\end{array}$ & $\begin{array}{l}\text { Synchro- } \\
\text { nous }\end{array}$ & $Y$ & $\begin{array}{l}\text { R. RCC dx in subse- } \\
\text { quent work up }\end{array}$ & $\begin{array}{l}\text { Liver, lungs, medi- } \\
\text { astinum, adrenal }\end{array}$ & $\begin{array}{l}\text { FNA non- } \\
\text { diagnostic }\end{array}$ & Palliative radiotherapy & $\begin{array}{l}\text { Positive for } \\
\text { vimentin } \\
\text { and keratin. } \\
\text { Negative for } \\
\text { CEA. }\end{array}$ \\
\hline $\begin{array}{l}\text { Gunbay } \\
\text { et. al. }\end{array}$ & 1989 & 60 & M & $\begin{array}{l}\text { Painful mass on } \mathrm{L} \text {. } \\
\text { and numbness of } \\
\mathrm{L} \text {. commisure of lip } \\
\text { x1mo. }\end{array}$ & $\begin{array}{l}\text { Metachro- } \\
\text { nous }\end{array}$ & $\mathrm{N}$ & $\begin{array}{l}\text { L. nephrectomy for } \\
\text { RCC } 2 \text { years prior }\end{array}$ & Not discussed & $\begin{array}{l}\text { Not men- } \\
\text { tioned }\end{array}$ & $\begin{array}{l}\text { Total parotidectomy } \\
\text { of mass extending into } \\
\text { pterygomaxillary fossa } \\
\mathrm{w} / \text { erosion of condylar } \\
\text { process of the mandible } \\
\text { and the TMJ }\end{array}$ & $\begin{array}{l}\text { None men- } \\
\text { tioned }\end{array}$ \\
\hline \multirow[t]{2}{*}{$\begin{array}{l}\text { Coppa } \\
\text { et. al. }\end{array}$} & 1990 & 42 & M & $\begin{array}{l}\text { Mass on L. } x 3 \\
\text { years, pulsatile } \\
\text { x1 year, tinnitus } \\
\text { x1 mo. }\end{array}$ & $\begin{array}{l}\text { Synchro- } \\
\text { nous }\end{array}$ & $\mathrm{Y}$ & $\begin{array}{l}\text { R. RCC dx in subse- } \\
\text { quent work up }\end{array}$ & $\begin{array}{l}\text { Perirenal lymph } \\
\text { nodes }\end{array}$ & $\begin{array}{l}\text { Not men- } \\
\text { tioned }\end{array}$ & $\begin{array}{l}\text { Superficial parotidec- } \\
\text { tomy } \mathrm{w} / \text { facial nerve } \\
\text { preservation }\end{array}$ & $\begin{array}{l}\text { None men- } \\
\text { tioned }\end{array}$ \\
\hline & & 55 & M & $\begin{array}{l}\text { Painful mass } x 6 \\
\text { weeks on R., bruit }\end{array}$ & $\begin{array}{l}\text { Metachro- } \\
\text { nous }\end{array}$ & $\mathrm{N}$ & $\begin{array}{l}\text { L. nephrectomy for } \\
\text { RCC } 7 \text { years prior }\end{array}$ & $\begin{array}{l}\text { Lungs, axillary } \\
\text { lymph nodes }\end{array}$ & $\begin{array}{l}\text { Not men- } \\
\text { tioned }\end{array}$ & $\begin{array}{l}\text { R. external carotid liga- } \\
\text { tion and multiple partial } \\
\text { parotid resections for } \\
\text { recurrent bleeding. }\end{array}$ & $\begin{array}{l}\text { None men- } \\
\text { tioned }\end{array}$ \\
\hline $\begin{array}{l}\text { Pisani } \\
\text { et. al. }\end{array}$ & 1990 & 59 & M & Mass x $2 \mathrm{mo}$. on $\mathrm{L}$. & $\begin{array}{l}\text { Synchro- } \\
\text { nous }\end{array}$ & $\mathrm{Y}$ & $\begin{array}{l}\text { L. RCC } d x \text { in subse- } \\
\text { quent work up }\end{array}$ & $\begin{array}{l}\text { Cerebellar, ver- } \\
\text { tebral }\end{array}$ & $\begin{array}{l}\text { Not men- } \\
\text { tioned }\end{array}$ & $\begin{array}{l}\text { Superficial parotidec- } \\
\text { tomy w/ preservation } \\
\text { of the facial nerve } \\
\text { followed by subsequent } \\
\text { hemorrhage and several } \\
\text { excisions for recurrent } \\
\text { disease }\end{array}$ & $\begin{array}{l}\text { None men- } \\
\text { tioned }\end{array}$ \\
\hline $\begin{array}{l}\text { Sarangi } \\
\text { et. al. }\end{array}$ & 1991 & 71 & $M$ & $\begin{array}{l}\text { Mass in L. parotid } \\
\text { x3mo at presenta- } \\
\text { tion, R. parotid } \\
\text { mass presented } 4 \\
\text { mo. later. }\end{array}$ & $\begin{array}{l}\text { Synchro- } \\
\text { nous }\end{array}$ & $Y$ & $\begin{array}{l}\text { R. RCC dx in subse- } \\
\text { quent work up }\end{array}$ & $\begin{array}{l}\text { None at time of } \\
\text { parotid presenta- } \\
\text { tions, later mets to } \\
\text { distal radius. }\end{array}$ & $\begin{array}{l}\text { Not men- } \\
\text { tioned }\end{array}$ & $\begin{array}{l}\text { L. superficial parotidec- } \\
\text { tomy followed by R. } \\
\text { superficial parotidec- } \\
\text { tomy. }\end{array}$ & $\begin{array}{l}\text { None men- } \\
\text { tioned }\end{array}$ \\
\hline
\end{tabular}


Lawlor et al. Journal of Cancer Therapeutics \& Research 2012,

http://www.hoajonline.com/journals/pdf/2049-7962-1-15.pdf

doi: 10.7243/2049-7962-1-15

\begin{tabular}{|c|c|c|c|c|c|c|c|c|c|c|c|}
\hline Author & Year & Age & Sex & Presenting symptom & $\begin{array}{l}\text { Synchro- } \\
\text { nous or } \\
\text { metachro- } \\
\text { nous }\end{array}$ & $\begin{array}{l}\text { First } \\
\text { sign } \\
\text { of } \\
\text { RCC? }\end{array}$ & RCC & $\begin{array}{l}\text { Other simultaneous } \\
\text { mets? }\end{array}$ & $\begin{array}{l}\text { Fine Needle } \\
\text { aspiration }\end{array}$ & Treatment & $\begin{array}{l}\text { Immunohisto- } \\
\text { chemistry }\end{array}$ \\
\hline $\begin{array}{l}\text { Ravi et. } \\
\text { al. }\end{array}$ & 1992 & 55 & $\mathrm{~F}$ & $\begin{array}{l}\text { Bilateral masses } \\
\text { x3mo. }\end{array}$ & $\begin{array}{l}\text { Metachro- } \\
\text { nous }\end{array}$ & $\mathrm{N}$ & $\begin{array}{l}\text { R. nephrectomy for } \\
\text { RCC } 7 \text { years prior }\end{array}$ & Not discussed & $\begin{array}{l}\text { Not men- } \\
\text { tioned }\end{array}$ & $\begin{array}{l}\text { Superficial parotidecto- } \\
\text { mies } \mathrm{w} / \text { preservation of } \\
\text { the facial nerves on two } \\
\text { separate occasions }\end{array}$ & $\begin{array}{l}\text { None men- } \\
\text { tioned }\end{array}$ \\
\hline $\begin{array}{l}\text { Borghi } \\
\text { et. al. }\end{array}$ & 1995 & 63 & M & Mass $x 1$ year on $R$. & $\begin{array}{l}\text { Synchro- } \\
\text { nous }\end{array}$ & Y & $\begin{array}{l}\text { L. RCC dx in subse- } \\
\text { quent work up }\end{array}$ & Liver, pancreas & $\begin{array}{l}\text { FNA non- } \\
\text { diagnostic }\end{array}$ & Partial parotidectomy & $\begin{array}{l}\text { Positive for } \\
\text { vimentin, } \\
\text { CAM5.2, AE1. } \\
\text { Negative for } \\
\text { S-100, CEA. }\end{array}$ \\
\hline $\begin{array}{l}\text { Sykes et. } \\
\text { al. }\end{array}$ & 1995 & 59 & M & $\begin{array}{l}\text { Mass } \times 3 \text { weeks } \\
\text { on } \mathrm{L} \text {. }\end{array}$ & $\begin{array}{l}\text { Synchro- } \\
\text { nous }\end{array}$ & Y & $\begin{array}{l}\text { L. RCC dx in subse- } \\
\text { quent work up }\end{array}$ & $\begin{array}{l}\text { Perirenal lymph } \\
\text { nodes }\end{array}$ & $\begin{array}{l}\text { FNA non- } \\
\text { diagnostic }\end{array}$ & $\begin{array}{l}\text { Superficial parotidec- } \\
\text { tomy } \mathrm{w} / \text { preservation of } \\
\text { the facial nerve }\end{array}$ & $\begin{array}{l}\text { None men- } \\
\text { tioned }\end{array}$ \\
\hline $\begin{array}{l}\text { Gango- } \\
\text { padhyay } \\
\text { et. al. }\end{array}$ & 1998 & 48 & M & Mass x3mo. on L. & $\begin{array}{l}\text { Synchro- } \\
\text { nous }\end{array}$ & $\mathrm{Y}$ & $\begin{array}{l}\text { L. RCC dx in subse- } \\
\text { quent work up }\end{array}$ & R. adrenal & $\begin{array}{l}\text { FNA non- } \\
\text { diagnostic }\end{array}$ & $\begin{array}{l}\text { Superficial parotidec- } \\
\text { tomy }\end{array}$ & $\begin{array}{l}\text { None men- } \\
\text { tioned }\end{array}$ \\
\hline $\begin{array}{l}\text { Vara et. } \\
\text { al. }\end{array}$ & 1998 & 50 & M & $\begin{array}{l}\text { Painful mass } \times 2 \mathrm{mo} \text {. } \\
\text { on } \mathrm{L} \text {. }\end{array}$ & $\begin{array}{l}\text { Metachro- } \\
\text { nous }\end{array}$ & $\mathrm{N}$ & $\begin{array}{l}\text { R. nephrectomy } 5 \\
\text { years prior }\end{array}$ & Not discussed & $\begin{array}{l}\text { Not men- } \\
\text { tioned }\end{array}$ & $\begin{array}{l}\text { Total parotidectomy w/ } \\
\text { preservation of facial } \\
\text { nerve, locally recurrent } \\
\text { in } 7 \text { mo., repeat excision } \\
\mathrm{w} / \text { radiotherapy }\end{array}$ & $\begin{array}{l}\text { None men- } \\
\text { tioned }\end{array}$ \\
\hline $\begin{array}{l}\text { Adil et. } \\
\text { al. }\end{array}$ & 1999 & 52 & M & Mass on R. & $\begin{array}{l}\text { Synchro- } \\
\text { nous }\end{array}$ & $\mathrm{N}$ & $\begin{array}{l}\text { L. nephrectomy for } \\
\text { RCC } 5 \text { mo. prior }\end{array}$ & $\begin{array}{l}\text { Post-auricular } \\
\text { lymph nodes at } \\
\text { time of parotid } \\
\text { presentation, later } \\
\text { para-aortic, ingui- } \\
\text { nal, and cervical } \\
\text { lymph nodes. }\end{array}$ & $\begin{array}{l}\text { Not men- } \\
\text { tioned }\end{array}$ & $\begin{array}{l}\text { Mass excision, neck } \\
\text { dissection }\end{array}$ & $\begin{array}{l}\text { None men- } \\
\text { tioned }\end{array}$ \\
\hline $\begin{array}{l}\text { Kundu } \\
\text { et. al. }\end{array}$ & 2001 & 61 & M & $\begin{array}{l}\text { R. facial weakness } \\
\text { and post-auricular } \\
\text { pain, R. mass on } \\
\text { physical exam. }\end{array}$ & $\begin{array}{l}\text { Synchro- } \\
\text { nous }\end{array}$ & $\mathrm{Y}$ & $\begin{array}{l}\text { L. RCC dx in subse- } \\
\text { quent work up }\end{array}$ & $\begin{array}{l}\text { R. adrenal, bone, } \\
\text { skin, pulmonary, } \\
\text { cerebral }\end{array}$ & $\begin{array}{l}\text { FNA non- } \\
\text { diagnostic }\end{array}$ & $\begin{array}{l}\text { Radiotherapy, pain } \\
\text { relief, hospice }\end{array}$ & $\begin{array}{l}\text { None men- } \\
\text { tioned }\end{array}$ \\
\hline $\begin{array}{l}\text { Park et. } \\
\text { al. }\end{array}$ & 2002 & 83 & $\mathrm{~F}$ & Mass x $2 \mathrm{mo}$. on $\mathrm{L}$. & $\begin{array}{l}\text { Metachro- } \\
\text { nous }\end{array}$ & $\mathrm{N}$ & $\begin{array}{l}\text { L. nephrectomy for } \\
\text { RCC } 10 \text { years prior }\end{array}$ & None & $\begin{array}{l}\text { FNA diag- } \\
\text { nostic }\end{array}$ & $\begin{array}{l}\text { Superficial parotidec- } \\
\text { tomy with preservation } \\
\text { of the facial nerve }\end{array}$ & $\begin{array}{l}\text { Positive for } \\
\text { vimentin } \\
\text { and keratin. } \\
\text { Negative for } \\
\text { CEA. }\end{array}$ \\
\hline $\begin{array}{l}\text { Seijas } \\
\text { et. al. }\end{array}$ & 2005 & 67 & M & Mass x4mo. on L. & $\begin{array}{l}\text { Synchro- } \\
\text { nous }\end{array}$ & Y & $\begin{array}{l}\text { R. RCC dx in subse- } \\
\text { quent work up }\end{array}$ & $\begin{array}{l}\text { L. adrenal at } \\
\text { time of parotid } \\
\text { presentation, later } \\
\text { lungs, R. adrenal, } \\
\text { retroparitoneal } \\
\text { lymph nodes }\end{array}$ & $\begin{array}{l}\text { FNA non- } \\
\text { diagnostic }\end{array}$ & $\begin{array}{l}\text { Superficial parotidec- } \\
\text { tomy with preservation } \\
\text { of the facial nerve }\end{array}$ & $\begin{array}{l}\text { Positive for } \\
\text { CAM5.2, } \\
\text { AE3, vimen- } \\
\text { tin. Negative } \\
\text { for S-100, } \\
\text { CK7, CK20, } \\
\text { EMA. }\end{array}$ \\
\hline \multirow[t]{2}{*}{$\begin{array}{l}\text { Mrena } \\
\text { et. al. }\end{array}$} & 2008 & 58 & $\mathrm{~F}$ & Tender mass on $\mathrm{R}$. & $\begin{array}{l}\text { Synchro- } \\
\text { nous }\end{array}$ & $\mathrm{Y}$ & $\begin{array}{l}\text { R. RCC dx in subse- } \\
\text { quent work up }\end{array}$ & $\begin{array}{l}\text { None at time of } \\
\text { presentation, R. } \\
\text { shoulder mets } \\
\text { later identified. }\end{array}$ & $\begin{array}{l}\text { FNA non- } \\
\text { diagnostic }\end{array}$ & $\begin{array}{l}\text { Superficial parotidec- } \\
\text { tomy }\end{array}$ & $\begin{array}{l}\text { Positive for } \\
\text { CD10, PNRA, } \\
\text { vimentin. } \\
\text { Partially posi- } \\
\text { tive for pan- } \\
\text { cytokeratin } \\
\text { (AE1/AE3). } \\
\text { Negative for } \\
\text { calponin, } \\
\text { S-100, and } \\
\text { cytokera- } \\
\text { tin-7. }\end{array}$ \\
\hline & & 76 & $\mathrm{~F}$ & $\begin{array}{l}\text { R. mass on physical } \\
\text { exam }\end{array}$ & $\begin{array}{l}\text { Metachro- } \\
\text { nous }\end{array}$ & $\mathrm{N}$ & $\begin{array}{l}\text { L. nephrectomy for } \\
\text { RCC } 9 \text { years prior }\end{array}$ & $\begin{array}{l}\text { Contralateral kid- } \\
\text { ney, lung, bone }\end{array}$ & $\begin{array}{l}\text { FNA non- } \\
\text { diagnostic }\end{array}$ & Not described & $\begin{array}{l}\text { Positive for } \\
\text { CD10, PNRA, } \\
\text { vimentin. } \\
\text { Negative for } \\
\text { cytokera- } \\
\text { tin-7. }\end{array}$ \\
\hline
\end{tabular}


Lawlor et al. Journal of Cancer Therapeutics \& Research 2012,

http://www.hoajonline.com/journals/pdf/2049-7962-1-15.pdf

\begin{tabular}{|c|c|c|c|c|c|c|c|c|c|c|c|}
\hline Author & Year & Age & Sex & Presenting symptom & $\begin{array}{l}\text { Synchro- } \\
\text { nous or } \\
\text { metachro- } \\
\text { nous }\end{array}$ & $\begin{array}{l}\text { First } \\
\text { sign } \\
\text { of } \\
\text { RCC? }\end{array}$ & RCC & $\begin{array}{l}\text { Other simultaneous } \\
\text { mets? }\end{array}$ & $\begin{array}{l}\text { Fine Needle } \\
\text { aspiration }\end{array}$ & Treatment & $\begin{array}{l}\text { Immunohisto- } \\
\text { chemistry }\end{array}$ \\
\hline & & 62 & M & $\begin{array}{l}\text { L. mass for a few } \\
\text { years }\end{array}$ & $\begin{array}{l}\text { Metachro- } \\
\text { nous }\end{array}$ & $\mathrm{N}$ & $\begin{array}{l}\text { R. nephrectomy for } \\
\text { RCC } 5 \text { years prior }\end{array}$ & None & $\begin{array}{l}\text { FNA non- } \\
\text { diagnostic }\end{array}$ & $\begin{array}{l}\text { Superficial parotidec- } \\
\text { tomy and selective neck } \\
\text { dissection (II-III) }\end{array}$ & $\begin{array}{l}\text { Positive for } \\
\text { CD10, vimen- } \\
\text { tin, CAM5.2. } \\
\text { Partially posi- } \\
\text { tive for CK-8 } \\
\text { and CK-18. } \\
\text { Negative for } \\
\text { cytokera- } \\
\text { tin-7. }\end{array}$ \\
\hline $\begin{array}{l}\text { Spre- } \\
\text { afico et. } \\
\text { al. }\end{array}$ & 2008 & 67 & M & $\begin{array}{l}\text { R.mass on physical } \\
\text { exam }\end{array}$ & $\begin{array}{l}\text { Metachro- } \\
\text { nous }\end{array}$ & $\mathrm{N}$ & $\begin{array}{l}\text { R. nephrectomy for } \\
\text { RCC } 18 \text { mo. prior }\end{array}$ & $\begin{array}{l}\text { Ipsilateral cervical } \\
\text { lymph nodes }\end{array}$ & $\begin{array}{l}\text { FNA diag- } \\
\text { nostic }\end{array}$ & $\begin{array}{l}\text { Total parotidectomy and } \\
\text { selective neck dissection } \\
\text { (I-V) w/removal of large } \\
\text { portion of masseter and } \\
\text { inferior facial nerve }\end{array}$ & $\begin{array}{l}\text { None men- } \\
\text { tioned }\end{array}$ \\
\hline $\begin{array}{l}\text { Wayne } \\
\text { et. al. }\end{array}$ & 2010 & 61 & $\mathrm{~F}$ & $\begin{array}{l}\text { L. mass on physical } \\
\text { exam }\end{array}$ & $\begin{array}{l}\text { Metachro- } \\
\text { nous }\end{array}$ & Y & No primary & Pancreas, skin & $\begin{array}{l}\text { Not men- } \\
\text { tioned }\end{array}$ & $\begin{array}{l}\text { Superficial parotidec- } \\
\text { tomy }\end{array}$ & $\begin{array}{l}\text { Positive } \\
\text { for PNRA, } \\
\text { vimentin, } \\
\text { CD10. }\end{array}$ \\
\hline $\begin{array}{l}\text { Deeb et. } \\
\text { al. }\end{array}$ & 2010 & 82 & M & $\begin{array}{l}\text { R. mass } \times 1.5 \text { years, } \\
\text { pain }\end{array}$ & $\begin{array}{l}\text { Metachro- } \\
\text { nous }\end{array}$ & $\mathrm{N}$ & $\begin{array}{l}\text { Partial nephrectomy } \\
\text { for RCC } 19 \text { years prior, } \\
\text { completion nephrec- } \\
\text { tomy } 4 \text { years prior }\end{array}$ & Not discussed & $\begin{array}{l}\text { FNA non- } \\
\text { diagnostic }\end{array}$ & Total parotidectomy & $\begin{array}{l}\text { None men- } \\
\text { tioned }\end{array}$ \\
\hline
\end{tabular}

the first sign of renal disease. Review of the cases presented in the literature suggests the most likely presentation is of an asymptomatic, unilateral parotid mass. Associated pain, palpable pulse, bruit, tinnitus, and numbness have been reported but are less common. Somewhat surprisingly, facial nerve weakness is extremely uncommon with only one documented case in the literature [14]. Although most cases of RCC are sporadic, hereditary forms (such as seen with associated with Von Hippel-Lindau disease) have been described and may require screening in high-risk populations.

Fine needle aspiration did not yield a diagnosis all but 3 of the cases reviewed. In this scenario, FNA is commonly non-diagnostic and carries a risk of false positive results. Even if a clear cell tumor is identified on FNA, differentiating the cells from a primary parotid tumor often requires immunohistochemical staining by an experienced pathologist. Differential diagnosis of clear cell tumors of the parotid gland includes clear cell variants of pleomorphic adenoma, myoepithelial carcinoma, epithelial-myoepithelial carcinoma, acinic cell carcinoma, adenocarcinoma of the salivary glands, hyalinizing clear cell carcinoma, mucoepidermoid carcinoma, oncocytoma, odontogenic carcinoma, and metastatic disease $[13,38]$. Clear cells can also result as artifact in slide preparation.

Immunohistochemistry can be used to distinguish RCC from other clear cell carcinomas of the parotid gland. RCC is known to stain positively for CD10 and vimentin, which were the utilized in the diagnosis of our patient [12]. CD10, also known as common acute lymphoblastic leukemia antigen (CALLA) or neprilysin, is a zinc-dependent metalloproteinase enzyme. Vimentin is an intermediate filament. Both are membranous stains that illuminate the cytoplasmic membrane when positive. CEA is more classically positive in clear cell tumors of parotid origin [12].
Appropriate management options for patients with parotid metastases from renal cell carcinoma is best determined by a multidisciplinary team that includes Otolaryngology, Medical Oncology, and Urology. Therapy for primary renal cell carcinoma ranges from radical or partial nephrectomy [4] to cytoreductive therapy (cryoablation and radiofrequency ablation) and is dependent upon the stage, grade and size of the presenting tumor. Isolated parotid metastasis is also ideally managed by local excision with disease-free margins with facial nerve preservation (when possible). Of the 27 cases in the literature that were managed with local excision, 3 experienced local recurrences $[16,24,29]$. Recurrence occurred following superficial parotidectomies in all 3 cases, with a disease-free interval of 18 months to 4 years. Two of those patients had untreated renal primaries at the time of disease recurrence [24,29].

Prognosis for patients with parotid metastasis from RCC is difficult to predict due to the limited number of cases reported in the literature. The most important prognostic factor for RCC is pathologic stage. Distant metastasis carries a 1 -year survival of 50\%, 5-year survival of 5 to $30 \%$, and 10 -year survival of 0 to $5 \%$. The presentation of an isolated parotid metastasis is favorable to multiple site distant metastatic spread of disease. Metachronous disease presentation suggests a less aggressive presentation [4]. Review of the literature demonstrated that greater than $50 \%$ of the patients found to have parotid RCC had other sites of metastasis. This highlights the need for a thorough metastatic work-up and a collaborative team approach for systemic renal cell carcinoma. Metastatic evaluation includes a chest radiograph, abdominal and pelvic $\mathrm{CT}$ or MRI, and liver function tests. Bone scan and chest $\mathrm{CT}$ can be reserved for patients with elevated alkaline phosphatase and abnormal chest radiographs, respectively [4]. 


\section{Conclusions}

Renal cell carcinoma (RCC) is a neoplasm with a unique metastatic potential to spread to nearly every organ system in the body. While RCC should not be high on the differential for all parotid gland masses, it should be considered when patients have a history of RCC and when FNA identifies a clear cell tumor. A thorough work-up should be performed, as solitary parotid metastases are rare in the literature and other occult sites may coexist. Isolated parotid disease is best managed with complete surgical excision and facial nerve preservation is usually possible.

\section{Competing interests}

The Authors declare that they have no competing interests.

\section{Author contributions}

$\mathrm{CL}$ was involved in the paper's conception, acquisition of data, drafting and revising of the manuscript. RW was involved in the paper's conception, revising and final approval of the manuscript. Both authors read and approved of the final version of the paper.

\section{Acknowledgments}

We would like to thank Tobi Quinto, MD and Rolf Pfannl, MD from Tufts Medical Center Department of Pathology for their assistance.

\section{Publication history}

Received: 31-May-2012 Revised: 21-June-2012

Accepted: 12-July-2012 Published: 14-July-2012

\section{References}

1. Spiro RH: Diagnosis and pitfalls in the treatment of parotid tumors. Semin Surg Oncol 1991; 7;(1.);20-4. | Article | PubMed

2. Bron LP, Traynor SJ, McNeil EB, O’Brien CJ: Primary and metastatic cancer of the parotid: comparison of clinical behavior in $\mathbf{2 3 2}$ cases. Laryngoscope 2003; 113;(6.);1070-5. | Article | PubMed

3. Nuyens M, Schupbach J, Stauffer E, Zbaren P: Metastatic disease to the parotid gland. Otolaryngol Head Neck Surg 2006; 135;(6.);844-8. I Article | PubMed

4. Campbell SC, Lane BR. Malignant renal tumors. In: Campbell-Walsh Urology, 10th Edition. Alan J. Wein, Ed. Philadelphia, PA: Saunders/Elsevier; 2007:1413-1474.

5. Park YW, Hlivko TJ: Parotid gland metastasis from renal cell carcinoma. Laryngoscope 2002; 112;(3.);453-6. I Article I PubMed

6. Bernicker EH, Khuri FR, Ellerhorst JA, et al. A case series of 65 patients with renal cell cancer presenting with metastasis to the head and neck region. Am Soc Clin Oncol Proc 1997;16:A1171.

7. Patey $D H$, Thackray $A C$, Keeling $D H$ : Malignant disease of the parotid. Br J Cancer 1965; 19;(4.);712-37. | PubMed Abstract | PubMed Full Text

8. Deeb R, Zhang Z, Kini S, Ghanem T: Metastatic renal cell carcinoma to the parotid gland presenting 19 years after nephrectomy: case report and review of literature. Laryngoscope 2010; 120 Suppl 4;(S128. | Article I PubMed

9. Wayne M, Wang W, Bratcher J, Cumani B, Kasmin F, Cooperman A: Renal cell cancer without a renal primary. World J Surg Oncol 2010; 8;(18. | Article | PubMed Abstract | PubMed Full Text

10. Spreafico R, Nicoletti G, Ferrario F, Scanziani R, Grasso M: Parotid metastasis from renal cell carcinoma: a case report and review of the literature. Acta Otorhinolaryngol Ital 2008; 28;(5.);266-8. I PubMed Abstract | PubMed Full Text

11. Mrena R, Leivo I, Passador-Santos F, Hagstrom J, Makitie AA: Histopathological findings in parotid gland metastases from renal cell carcinoma. Eur Arch Otorhinolaryngol 2008; 265;(9.);1005-9. | Article

\section{PubMed}

12. Andreadis D, Nomikos A, Barbatis C: Metastatic renal clear cell carcinoma in the parotid gland: a study of immunohistochemical profile and cell adhesion molecules (CAMs) expression in two cases. Pathol Oncol Res 2007; 13;(2.);161-5. | Article | PubMed

13. Seijas BP, Franco FL, Sastre RM, Garcia AA, Lopez-Cedrun Cembranos $\mathrm{JL}$ : Metastatic renal cell carcinoma presenting as a parotid tumor. Oral Surg Oral Med Oral Pathol Oral Radiol Endod 2005; 99;(5.);554-7. | Article I PubMed

14. Kundu S, Eynon-Lewis NJ, Radcliffe GJ: Extensive metastatic renal cell carcinoma presenting as facial nerve palsy. J Laryngol Otol 2001; 115;(6.);488-90. | Article | PubMed

15. Adil G, Murat D, Ayhan O, Ozgur TM, Ibrahim Y, Fuat PA, et al.: Renal cell carcinoma metastasis to the parotid gland. BJU Int 1999; 83;(7.);861-2. | Article | PubMed

16. Vara A, Madrigal B, Perez del Rio MJ, Diaz A, Mateos A, Sales C: Parotid metastasis from renal clear cell adenocarcinoma. An unusual site for metastasis. Urol Int 1998; 61;(3.);196-8. | Article | PubMed

17. Gangopadhyay K, Abuzeid MO, Martin JM, Saleem M: Metastatic renal cell carcinoma of the parotid gland presenting as a neck mass. Int J Clin Pract 1998; 52;(3.);196-8. | PubMed

18. Malata CM, Camilleri IG, McLean NR, Piggot TA, Kelly CG, Chippindale $\mathrm{AJ}$, et al.: Malignant tumours of the parotid gland: a 12-year review. $\mathrm{Br}$ J Plast Surg 1997; 50;(8.);600-8. I PubMed

19. Sykes TCF, Patel A, Archer D, Fisher C, Hendry WF: Parotid metastasis from renal cell carcinoma. British Journal of Urology 1995; 76;(3.);3989. | Article

20. Borghi L, Bianchini E, Ballotta MR, Reale D: Metastatic renal cell carcinoma presenting as a parotid tumor: a case report. Pathologica 1995; 87;(2.);168-70. | PubMed

21. Stanley MW, Bardales RH, Farmer CE, Frierson HF, Jr., Suhrland M, Powers $\mathrm{CN}$, et al.: Primary and metastatic high-grade carcinomas of the salivary glands: a cytologic-histologic correlation study of twenty cases. Diagn Cytopathol 1995; 13;(1.);37-43. | Article | PubMed

22. Ravi R, Tongaonkar HB, Kulkarni JN, Kamat MR: Synchronous bilateral parotid metastases from renal cell carcinoma. A case report. Indian J Cancer 1992; 29;(1.);40-2. I PubMed

23. Sarangi $P P$, Hameed B: Bilateral parotid gland metastases from a hypernephroma. J R Coll Surg Edinb 1991; 36;(2.);128. | PubMed

24. Pisani $P$, Angeli G, Krengli M, Pia F: Renal carcinoma metastasis to the parotid gland. The Journal of Laryngology \& Otology 1990; 104;(04.);352-4. | Article

25. Coppa GF, Oszczakiewicz M: Parotid gland metastasis from renal carcinoma. Int Surg 1990; 75;(3.);198-202. I PubMed

26. Owens RM, Friedman CD, Becker SP: Renal cell carcinoma with metastasis to the parotid gland: case reports and review of the literature. Head Neck 1989; 11;(2.);174-8. | Article | PubMed

27. Günbay MU, Ceryan K, Küpelíjogülu AA: Metastatic renal carcinoma to the parotid gland. The Journal of Laryngology \& Otology 1989; 103;(04.);417-8. | Article

28. Melnick SJ, Amazon K, Dembrow V: Metastatic renal cell carcinoma presenting as a parotid tumor: a case report with immunohistochemical findings and a review of the literature. Hum Pathol 1989; 20;(2.);195-7. | Article | PubMed

29. Harrison DJ, McLaren K, Tennant W: A clear cell tumour of the parotid. The Journal of Laryngology \& Otology 1987; 101;(06.);633-5. | Article

30. Hessan $H$, Strauss $M$, Sharkey FE: Urogenital tract carcinoma metastatic to the head and neck. Laryngoscope 1986; 96;(12.);1352-6. | Article | PubMed

31. Seifert G, Hennings K, Caselitz J: Metastatic tumors to the parotid and submandibular glands--analysis and differential diagnosis of 108 cases. Pathol Res Pract 1986; 181;(6.);684-92. | Article | PubMed

32. Smits JG, Slootweg PJ: Renal cell carcinoma with metastasis to the submandibular and parotid glands. A case report. J Maxillofac Surg 1984; 
12;(5.);235-6. | Article | PubMed

33. Percival RC, Curt JR: Metastatic hypernephroma of the parotid gland. Postgrad Med J 1982; 58;(677.);167-8. | Article | PubMed

34. Sist TC, Jr., Marchetta FC, Milley PC: Renal cell carcinoma presenting as a primary parotid gland tumor. Oral Surg Oral Med Oral Pathol 1982; 53;(5.);499-502. | Article | PubMed

35. Kucan JO, Frank DH, Robson MC: Tumours metastatic to the parotid gland. British Journal of Plastic Surgery 1981; 34;(3.);299-301. I Article

36. Smith RL, Davis TS, Kennedy TJ, Graham WP, 3rd, Miller SH: Metastatic malignancies of the parotid gland. Am Fam Physician 1977; 16;(5.);13940. | PubMed

37. Oikarinen VJ, Calonius PEB, Sainio P. Metastatic tumors of the oral origin. Proc Finn Dent Soc. 1975;71:58-71.

38. Maiorano E, Altini M, Favia G: Clear cell tumors of the salivary glands, jaws, and oral mucosa. Semin Diagn Pathol 1997; 14;(3.);203-12. I PubMed

39. Gogus $C$, Kilic O, Tulunay O, Beduk Y: Solitary metastasis of renal cell carcinoma to the parotid gland $\mathbf{1 0}$ years after radical nephrectomy. Int J Urol 2004; 11;(10.);894-6. | Article | PubMed

\section{Citation:}

Lawlor CM and Wein RO: Parotid Metastasis of Renal Cell Carcinoma: A Case Report and Review of the Literature. journal of Cancer Therapeutics and Research 2012, 1:15.

http://dx.doi.org/10.7243/2049-7962-1-15 